\title{
Robust Genetic Algorithm and Fuzzy Inference Mechanism Embedded in Sliding-Mode Controller for Uncertain Underwater Robot
}

\author{
Cheng Siong Chin, Senior Member, IEEE and Wei Peng Lin
}

\begin{abstract}
The integration of inaccurate remotely-operated vehicle (ROV) model obtained by computational fluid dynamics for control is presented. Since the ROV is highly nonlinear and uncertain, a sliding-mode control (SMC) system using a direction-based genetic algorithm (GA) and fuzzy inference mechanism is used. The GA influences the right evolutionary step and direction of the SMC parameters subjected to uncertainties in the evolutionary process. The effectiveness of reducing the sensitivity of the proposed control scheme to model parameters and external disturbance is verified by simulations and sea trial. The results demonstrate the proposed controller performed better with less chattering in position responses than SMC without GA-Fuzzy optimization, fuzzy logic controller, and proportional integral derivative (PID).
\end{abstract}

Index Terms - Hydrodynamic parameters, RemotelyOperated Vehicle, Genetic Algorithm, Sliding-Mode Control, Fuzzy Logic.

\section{INTRODUCTION}

$\mathrm{M}$ MODELING and control techniques are essential in many engineering applications. The applications of numerical modeling techniques on marine vehicles such as an underwater robotic vehicle (URV) have been gradually increased in the last few decades. The remotely operated vehicle (ROV) is commonly used to carry out several tasks in deeper and riskier areas where divers are not possible. However, there are numbers of challenges in operating ROV precisely; unpredictable disturbances such as the sea current and wave during ROV operation and uncertain dynamic model for designing a typical guidance, navigation, and control system.

Unfortunately, the standard dynamics model of the ROV[1] used for inspection task [2] is not easy to obtain due to the complex-shaped design as compared to the streamlined components in an autonomous underwater vehicle (AUV) [3-5]. The latter exists empirical solutions to solve for the hydrodynamics parameters for model-based control purpose. Traditionally, the hydrodynamic parameters and underwater behavior of ROV are identified using a labbased experimental approach. However, this test method is costly, time-consuming and requiring test facilities and accurate scale prototype model. Computational fluid dynamic (CFD) technology has been commonly applied on AUV [6-7] to validate the hydrodynamic coefficients as compared to the empirical solutions. Another approach using system identification method such as adaptive least square [8] was applied on AUV to estimate the model's parameters for control. However, the method involved extensive sea trial before the model could be used for control. In addition, a numerical method was applied on a scale streamlined underwater vehicle [9] to predict the hydrodynamics parameters. Although it involved no sea trial, the range of errors resulted from the nonlinear least square curve fitting and experiment was more than 30\%[9] in the paper. As shown in the above examples, the prediction of a reasonably accurate set of hydrodynamic parameters for ROV is, therefore, difficult due to the openframe geometry and the hydrodynamics computation involved [10]. Hence, there is a need to have a controller to overcome the varying model uncertainties obtained from different modeling approaches.

Sliding mode control (SMC) is one of the robust controllers for controlling different dynamical systems with mentioned uncertainties. For example, sliding mode observer [11] was designed to estimate the sideslip and steering angle for a linear-parameter-varying system on the electric vehicle subjected to model uncertainties and external disturbances without dealing with control system design. The adaptive SMC [12] was used for the parallel robot with six prismatic actuators under the loss of actuator. However, the adaptive approach to tune the sliding mode controller parameters depend on different time-varying upper bounds (i.e., for the initial time and final time) of the uncertainties that are difficult to determine. Another approach of using SMC on an uncertain underwater robot [13] was proposed. The controller gains that affect the control stability and rate of convergent were tuned offline. The types of external uncertainties such as underwater current that is inherent to underwater robot were not defined. Other similar SMC application on different imprecise models can be found in [14] and [15]. In the above-mentioned literature, the automatic tuning of SMC parameters is not studied for the class of uncertain ROV system.

Instead of using the model-free approach based on the combination of Neural Network (NN) for modelling and $\mathrm{SMC}[16]$ for control that requires long computation training time, a robust SMC with online tuning of controller parameters via genetic algorithm (GA) and fuzzy logic to control an uncertain nonlinear ROV model obtained from CFD is proposed. There are few control algorithms based on one of more combination of Fuzzy, NN, Genetic Algorithm (GA) and SMC. The method to tune the SMC parameters will be examined. For example, the adaptive fuzzy-NN (FNN) [17] was designed to eliminate the chattering by tuning the SMC law online. The optimal FNN to learn the SMC law with a reconstructed-error vector was determined. The computation time taken to compute the control law for 
the robot manipulator can increase due to the training of the 4-layer FNN with a large number of neurons. The minimum bound of the reconstructed-error vector in the control law depends on the large sample size and number of neurons in the FNN. The similar concept but using type-2 fuzzy logic controllers based on SMC based learning algorithm was applied to control the yaw dynamics of an autonomous vehicle [18]. The results mentioned the robustness of the control approach without demonstrating how the model uncertainties and external disturbances (despite its presence in most system) can be modeled. An adaptive algorithm [19] to adjust the fuzzy parameter was used to cope with the bounded external disturbances only. But the common drawback of such adaptive based approach is the need to create a continuously parameterized number of candidate controllers that can pose some difficulty and challenges in the actual implementation of the number of controllers used.

Besides using FNN-SMC, GA optimization approach [20] was used to learn and classify the parameters. Identification of unmanned system using the GA was employed [21]. The application on control system design using a standard application example was shown. First, the fuzzy modeling method was used to model the cart-pole typed inverted pendulum (CPIP) system [22] followed by the designing nonlinear controller gains via GA. The stability of the fuzzymodel-based control can be guaranteed if the controller gains were chosen appropriately via linear matrix inequalities. Similar approach was applied for two-link robot arm (TRA) [23] but an improved GA [24] was used to design the feedback gain of the fuzzy controller to ensure the stability of the system. However, the model uncertainties and external disturbances that affect the stability and robustness of the control system design was not studied in the two papers [22-23]. In particular, the uncertain plant model subjected to perturbations was not included in the evolutionary procedure. Also, it was applied on application examples of the linked robot arm, and the inverted pendulum system without validating with other complex systems such as ROV in water.

In this paper, the proposed fuzzy-GA based SMC will address the current stability of the GA optimization on the controller design of an uncertain model. A complex mechatronic systems that sufficiently represent the nonlinear model with uncertainties and external disturbance such as the ROV was used [10]. The fuzzy inference mechanism will be used on the crossover and mutation in GA to influence the correct evolutionary step and direction under the different model and external perturbation. The SMC will define the GA as a direction-based GA that is distinct from the conventional GA using random numbers and weightings. In addition, the stability of the GA optimization is achieved using the robust SMC. The numerical model of ROV consisting of hydrodynamic damping and added mass coefficients will be included in the evolutionary procedure.

In summary, the proposed evolutionary procedure applied on the plant model subjected to perturbations are not considered in most real-time GA process. The use of the direction-based GA and fuzzy inference mechanism on SMC leads to a more efficient architecture for tuning SMC as it influences the direction of the SMC's gain for improved stability with less chattering phenomena. The stability can be consequentially ensured by the SMC and fuzzy-based evolutionary procedure during the entire control process.

The following summarized the main contributions. First, the integration of inaccurate ROV model obtained by computational fluid dynamic during the control system design process. Second, the use of robust SMC with the fuzzy-based evolutionary procedure on an actual model subjected to model perturbation and external uncertainties in the evolutionary process. Last, the SMC was integrated into GA to develop a real-time code GA control systems on a real ROV platform with good position responses with less chattering. A simulation and experimental results were given to show the effectiveness of the proposed approach.

The rest of the paper is organized as follows. The numerical modeling of ROV is given in Section II followed by robust control system designs involving SMC and fuzzybased evolutionary procedure in Section III. In Section IV, simulation and experimental results from sea trial are discussed. Lastly, the paper concludes in Section V.

\section{NUMERICAL MODELING OF ROV}

\section{A. ROV Dynamics and Kinematics}

The nonlinear ROV model consists of dynamics and kinematics. As shown in Fig. 1, two reference coordinates are used to describe the position of ROV in an earth-fixed or inertial frame coordinate $\left(\boldsymbol{\eta}=\left[\boldsymbol{\eta}_{1} \mid \boldsymbol{\eta}_{\mathbf{2}}\right]^{\mathrm{T}}=\left[\begin{array}{lllllll}x & y & z & \phi & \theta & \psi\end{array}\right]^{\mathrm{T}}\right)$ and the velocity body-fixed coordinate $\left(\mathbf{v}=\left[\mathbf{v}_{1} \mid \mathbf{v}_{2}\right]^{\mathrm{T}}=\left[\begin{array}{lllllll}u & v & w & p & q & r\end{array}\right]^{\mathrm{T}}\right)$ at the origin of ROV's center of gravity. The three principal axes are namely surge, sway and heave at the body of ROV as shown in Fig. 1. The actuated ROV system has six degrees of freedoms (DoFs).
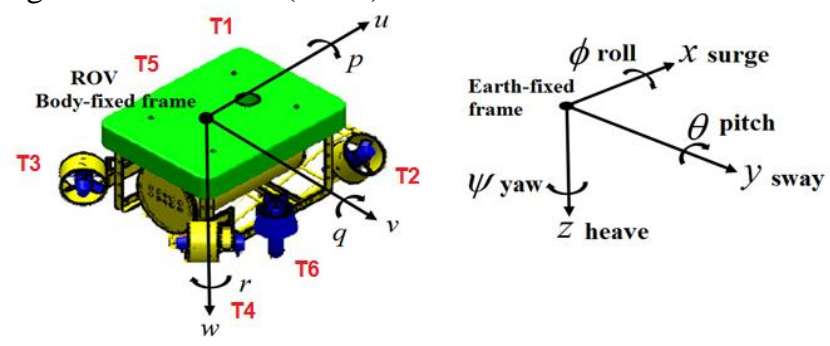

Fig. 1. Earth-fixed and body-fixed frame on ROV

The dynamics of ROV can be expressed as shown. The detailed derivations can be found in [1] and are omitted here.

$$
\mathbf{M} \dot{\mathbf{v}}+\mathbf{C}(\mathbf{v}) \mathbf{v}+\mathbf{D}(\mathbf{v})+\mathbf{g}(\boldsymbol{\eta})=\boldsymbol{\tau}
$$

where $\mathbf{V}$ is the body-fixed velocity vector, $\boldsymbol{\eta}$ is the earthfixed vector, $\mathbf{M}=\mathbf{M}_{\mathbf{R B}}+\mathbf{M}_{\mathbf{A}} \in \mathfrak{R}^{6 \times 6}$ is the sum of mass inertia matrix and added mass terms, $\mathbf{g}(\boldsymbol{\eta}) \in \mathfrak{R}^{6}$ is the gravitational and buoyancy vector, $\mathbf{C}(\mathbf{v})=\mathbf{C}_{\mathbf{R B}}(\mathbf{v})+\mathbf{C}_{\mathbf{A}}(\mathbf{v}) \in \mathfrak{R}^{6 \times 6}$ is the sum of Coriolis and centripetal matrix and added mass terms, $\mathbf{D}(\mathbf{v}) \in \mathfrak{R}^{6 \times 6}$ is the sum of linear, $\mathbf{D}_{\mathbf{L}} \in \mathfrak{R}^{6 \times 6}$ and quadratic damping matrix, $\mathbf{D}_{\mathbf{Q}} \in \mathfrak{R}^{6 \times 6}, \boldsymbol{\tau}=\left[\tau_{x} \tau_{y} \tau_{z} \tau_{\phi} \tau_{\theta} \tau_{\psi}\right]^{\mathrm{T}}=\mathbf{T} \mathbf{u} \in \mathfrak{R}^{6}$ is the forces and moments vector generated by the six thrusters. The thruster configuration matrix $\mathbf{T} \in \mathfrak{R}^{6 \times 6}$ defines the forces and moments allocations based on the layout of the thrusters on ROV platform. The T matrix is obtained by summing the 
forces in $x-y-z$ direction and taking the moment about $X-Y-Z$ axes. For example, the thrust generated by row 1 of $\mathbf{T}$ matrix can be obtained by the two thrusters, namely T3 and T4 (see the third and fourth column of (2)) are used to move the ROV in $x$-direction. The thrust generated by T3 and T4 in $x$ direction will give the respective value in the first row of (2). Similarly, T1 to T4 are used to actuate the ROV in the $y$-direction. The thrust generated by these thrusters in $y$ direction can be seen in the second row of (2). The negative sign indicates the opposite direction of motion. The same interpretation can be provided for the remaining DoFs.

$\mathbf{T}=\left[\begin{array}{c}T_{x} \\ T_{y} \\ T_{z} \\ T_{\phi} \\ T_{\theta} \\ T_{\psi}\end{array}\right]=\left[\begin{array}{cccccc}0 & 0 & \cos v & \cos v & 0 & 0 \\ -\cos \xi & \cos \xi & \sin v & -\sin v & 0 & 0 \\ \cos \xi & \cos \xi & 0 & 0 & 1 & 1 \\ 0.155 \cos \xi & -0.155 \cos \xi & 0 & 0 & -0.275 & 0.275 \\ 0.394 \cos \xi & 0.394 \cos \xi & 0.430 \cos \xi & 0.430 \cos \xi & -0.035 & -0.035 \\ -0.394 \sin \xi & 0.394 \sin \xi & -0.660 \sin \xi & 0.660 \sin \xi & 0 & 0\end{array}\right]$

where $v=45^{\circ}$ is the inclination angle for T3 and T4 while $\xi=45^{\circ}$ is the orientation angle for $\mathrm{T} 1$ and $\mathrm{T} 2$. Note that T5 and T6 are the two vertical thrusters shown in Fig. 1. The resulting $\mathbf{T}$ matrix can be computed as shown.

$$
\mathbf{T}=\left[\begin{array}{cccccc}
0 & 0 & 0.7070 & 0.7070 & 0 & 0 \\
-0.7070 & 0.7070 & 0.7070 & -0.7070 & 0 & 0 \\
0.7070 & 0.7070 & 0 & 0 & 1 & 1 \\
0.1099 & -0.1099 & 0 & 0 & -0.2750 & 0.2750 \\
0.2789 & 0.2789 & 0.3044 & 0.3044 & -0.0355 & -0.0355 \\
-0.2789 & 0.2789 & -0.4670 & 0.4670 & 0 & 0
\end{array}\right]
$$

The rigid-body mass and inertia matrix $\mathbf{M}_{\mathbf{R B}}$ are obtained through the computer-aided design (CAD) software where each mass and size of the components of the origin of ROV is modeled. It enables ROV's moments of inertia, I to be computed w.r.t. Centre of gravity. The total mass of the ROV is weighted using an actual weighing scale to check the accuracy of the CAD modeling. The ROV's weight in the three principal directions (i.e. surge, sway, and heave) is approximately $m=135 \mathrm{~kg}$ in the air as compared to the mass equals to $133.4 \mathrm{~kg}$ from the software.

$$
\mathbf{M}_{\mathbf{R B}}=\left[\begin{array}{cc}
m \mathbf{I}_{3} & 0 \\
0 & \mathbf{I}
\end{array}\right]=\left[\begin{array}{cccccc}
135 & 0 & 0 & 0 & 0 & 0 \\
0 & 135 & 0 & 0 & 0 & 0 \\
0 & 0 & 135 & 0 & 0 & 0 \\
0 & 0 & 0 & 2.51 & 0 & 0 \\
0 & 0 & 0 & 0 & 3.38 & -0.01 \\
0 & 0 & 0 & 0 & -0.01 & 1.73
\end{array}\right]
$$

where $\mathbf{I}_{3}$ is an identity matrix of size 3 and $\mathbf{I}$ is the mass moment of inertia of ROV.

The rigid-body Coriolis and centripetal matrix[1] describes the angular motion of the vehicle as follows.

$$
\mathbf{C}_{\mathrm{RB}}(\mathbf{v})=\left[\begin{array}{cc}
\mathbf{0}_{3 \times 3} & \mathrm{C}_{12}(\mathbf{v}) \\
-\mathbf{C}_{12}^{\mathrm{T}}(\mathbf{v}) & \mathrm{C}_{22}(\mathbf{v})
\end{array}\right]
$$

and

$$
\begin{gathered}
\mathbf{C}_{\mathbf{1 2}}(\mathbf{v})=\left[\begin{array}{ccc}
m\left(y_{G} q+z_{G} r\right) & -m\left(x_{G} q-w\right) & -m\left(x_{G} r+v\right) \\
-m\left(y_{G} p+w\right) & m\left(z_{G} r+x_{G} p\right) & -m\left(y_{G} r-u\right) \\
-m\left(z_{G} p-v\right) & -m\left(z_{G} q+u\right) & m\left(x_{G} p+y_{G} q\right)
\end{array}\right] \\
\mathbf{C}_{22}(\mathbf{v})=\left[\begin{array}{ccc}
0 & -I_{y z} q-I_{x z} p+I_{z} r & I_{y z} r+I_{x y} p-I_{y} q \\
I_{y z} q+I_{x z} p-I_{z} r & 0 & -I_{x z} r-I_{x y} q+I_{x} p \\
-I_{y z} r-I_{x y} p+I_{y} q & I_{x z} r+I_{x y} q-I_{x} p & 0
\end{array}\right]
\end{gathered}
$$

where the component $m$ represents the mass of the vehicle (where $I_{x}, I_{y}, I_{z} I_{x y}, I_{x z}, I_{y z}$ represent the mass moments of inertia of ROV). The coordinates of the center of gravity of $\mathrm{ROV}$ are $\mathrm{x}_{\mathrm{G}}, \mathrm{y}_{\mathrm{G}}$ and $\mathrm{z}_{\mathrm{G}}$. The resulting $\mathbf{C}_{\mathrm{RB}}(\mathbf{v})$ matrix becomes

$$
\mathbf{C}_{\mathbf{R B}}(\mathbf{v})=\left[\begin{array}{cccccc}
0 & 0 & 0 & 0 & 135 w & -135 v \\
0 & 0 & 0 & -135 w & 0 & 135 u \\
0 & 0 & 0 & 135 v & -135 u & 0 \\
0 & 135 w & -135 v & 0 & 0.01 q+1.73 r & -0.01 r-3.38 q \\
-135 w & 0 & 135 u & -0.01 q-1.73 r & 0 & 2.51 p \\
135 v & -135 u & 0 & 0.01 r+3.38 p & -2.51 p & 0
\end{array}\right]
$$

For three planes of symmetry on ROV, the added mass matrix [1] is a diagonal matrix with non-diagonal elements equal to zero.

$$
\mathbf{M}_{\mathbf{A}}=\operatorname{diag}\left\{\left[\begin{array}{llllll}
X_{\dot{u}} & Y_{\dot{v}} & Z_{\dot{w}} & K_{\dot{p}} & M_{\dot{q}} & N_{\dot{r}}
\end{array}\right]\right\}
$$

where $X_{\dot{u}}$ is the added mass along $Y$-axis due to an acceleration in the $x$-direction, $Y_{\dot{v}}$ is the added mass along $Y$-axis due to an acceleration $\dot{v}$ in $y$-direction and similar interpretation are applied to other parameters in the matrix.

The correspondence added Coriolis, and centripetal matrix [1] using the same added mass values and definitions in (9) can be expressed as

$$
\mathbf{C}_{\mathbf{A}}(\mathbf{v})=\left[\begin{array}{cccccc}
0 & 0 & 0 & 0 & -Z_{\dot{w}} w & Y_{\dot{v}} v \\
0 & 0 & 0 & Z_{\dot{w}} w & 0 & -X_{\dot{u}} u \\
0 & 0 & 0 & -Y_{\dot{v}} v & X_{\dot{u}} u & 0 \\
0 & -Z_{\dot{w}} w & Y_{\dot{v}} v & 0 & -N_{\dot{r}} r & M_{\dot{q}} q \\
Z_{\dot{w}} w & 0 & -X_{\dot{u}} u & N_{\dot{r}} r & 0 & -K_{\dot{p}} p \\
-Y_{\dot{v}} v & X_{\dot{u}} u & 0 & -M_{\dot{q}} q & K_{\dot{p}} p & 0
\end{array}\right]
$$

Both linear and nonlinear damping matrices have a diagonal structure as shown.

$$
\begin{aligned}
& \mathbf{D}_{\mathbf{L}}=\operatorname{diag}\left\{\left[\begin{array}{llllll}
X_{u} & Y_{v} & Z_{w} & K_{p} & M_{q} & N_{r}
\end{array}\right]\right\} \\
& \mathbf{D}_{\mathbf{Q}}=\operatorname{diag}\left\{\left[\begin{array}{llllll}
X_{u|u|} & Y_{v|v|} & Z_{w|w|} & K_{p|p|} & M_{q|q|} & N_{r|r|}
\end{array}\right]\right.
\end{aligned}
$$

where $X_{u}$ is the linear damping coefficient in $x$-direction and $X_{v|v|}$ is the quadratic damping coefficient in the $x$ direction. Similar interpretation is given to the remaining parameters in these matrices.

As the ROV is neutrally buoyant and the center of gravity coincides with the center of buoyancy, the gravitational and buoyancy vector $\mathbf{g}(\boldsymbol{\eta})$ becomes zero. The kinematics equation [1] is written as:

$$
\dot{\boldsymbol{\eta}}=\mathbf{J}\left(\boldsymbol{\eta}_{2}\right) \mathbf{v}=\left[\begin{array}{cc}
\mathbf{J}_{1}\left(\boldsymbol{\eta}_{2}\right) & 0 \\
0 & \mathbf{J}_{2}\left(\boldsymbol{\eta}_{2}\right)
\end{array}\right] \mathbf{v}
$$

where $\mathbf{J}\left(\boldsymbol{\eta}_{\mathbf{2}}\right)$ is the Euler transformation matrix that transforms earth-fixed frame coordinate into the body-fixed coordinate. The $\mathbf{J}_{\mathbf{1}}\left(\boldsymbol{\eta}_{\mathbf{2}}\right)$ and $\mathbf{J}_{\mathbf{2}}\left(\boldsymbol{\eta}_{\mathbf{2}}\right)$ are written as follows.

$\mathbf{J}_{\mathbf{1}}\left(\boldsymbol{\eta}_{2}\right)=\left[\begin{array}{ccc}\mathrm{c}(\psi) \mathrm{c}(\theta) & -\mathrm{s}(\psi) \mathrm{c}(\phi)+\mathrm{c}(\psi) \mathrm{s}(\theta) \mathrm{s}(\phi) & \mathrm{s}(\psi) \mathrm{s}(\phi)+\mathrm{c}(\psi) \mathrm{c}(\phi) \mathrm{s}(\theta) \\ \mathrm{s}(\psi) \mathrm{c}(\theta) & \mathrm{c}(\psi) \mathrm{c}(\phi)+\mathrm{s}(\phi) \mathrm{s}(\theta) \mathrm{s}(\psi) & -\mathrm{c}(\psi) \mathrm{s}(\phi)+\mathrm{s}(\theta) \mathrm{s}(\psi) \mathrm{c}(\theta) \\ -\mathrm{s}(\theta) & \mathrm{c}(\theta) \mathrm{s}(\phi) & \mathrm{c}(\theta) \mathrm{c}(\phi)\end{array}\right]$

$$
\mathbf{J}_{2}\left(\boldsymbol{\eta}_{2}\right)=\left[\begin{array}{ccc}
1 & \mathrm{~s}(\phi) \mathrm{t}(\theta) & \mathrm{c}(\phi) \mathrm{t}(\theta) \\
0 & \mathrm{c}(\phi) & -\mathrm{s}(\phi) \\
0 & \frac{\mathrm{s}(\phi)}{\mathrm{c}(\theta)} & \frac{\mathrm{c}(\phi)}{\mathrm{c}(\theta)}
\end{array}\right]
$$

where $\mathrm{s}=\sin (),. \mathrm{c}=\cos (),. \mathrm{t}=\tan ($.$) . Since the parameters$ in $\mathbf{J}$ can be singular, the attitude parameters are restricted to the admissible set, $\vartheta=(-\pi, \pi] \times(-\pi / 2, \pi / 2)$ $\times(-\pi, \pi] \subset \mathfrak{R}^{3}$. Notice that the parameters in the damping and added mass terms in (9) to (12) are determined using the computational fluid dynamic (CFD) software, and experiments as shown in next section. 


\section{B. ROV Model Validation}

The CFD software such as STAR-CCM+ ${ }^{\mathrm{TM}}$ and WAMIT $^{\mathrm{TM}}$ were used to determine the hydrodynamic damping and added mass parameters of ROV, respectively. Figure 2 illustrates the flow around ROV where the hydrodynamic damping forces and moment are obtained by integrating the pressure over different flow velocities around the vehicle using STAR-CCM+ ${ }^{\mathrm{TM}}$. The hydrodynamic added mass was subsequently computed by WAMIT $^{\mathrm{TM}}$ using the low-order panel method. The results of the hydrodynamic damping and added mass in six DoFs are tabulated in Table 1 and 2[10].

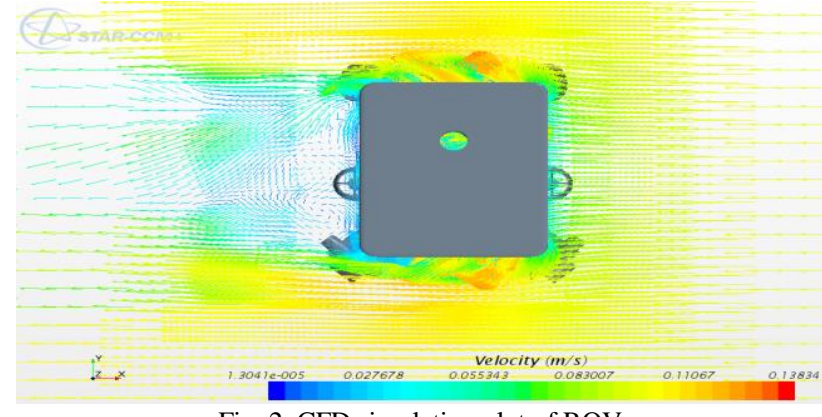

Fig. 2. CFD simulation plot of ROV

TABLE 1: HYDRODYNAMIC DAMPING AND ADDED MASS COEFFICIENTS FOR LINEAR DIRECTION

\begin{tabular}{|c|c|c|c|c|c|c|}
\hline Descriptions & \multicolumn{2}{|c|}{ Surge } & \multicolumn{2}{|c|}{ Sway } & \multicolumn{2}{|c|}{ Heave } \\
\hline $\begin{array}{l}\text { Damping } \\
\text { coefficients }\end{array}$ & $X_{u}$ & $X_{u|u|}$ & $Y_{v}$ & $Y_{v|v|}$ & $Z_{w}$ & $Z_{w|w|}$ \\
\hline $\begin{array}{l}\text { CFD: STAR } \\
\text { CCM+ }\end{array}$ & 3.22 & 105 & 3.29 & 139 & 5.68 & 273 \\
\hline Experiment & - & - & - & - & 3.78 & \\
\hline $\begin{array}{l}\text { CFD: Added } \\
\text { mass } \\
\text { coefficients }\end{array}$ & \multicolumn{2}{|c|}{$X_{\dot{u}}$} & \multicolumn{2}{|c|}{$Y_{\dot{v}}$} & \multicolumn{2}{|c|}{$Z_{\dot{w}}$} \\
\hline WAMIT & \multicolumn{2}{|c|}{20.4} & \multicolumn{2}{|c|}{53.4} & \multicolumn{2}{|c|}{126} \\
\hline Experiment & \multicolumn{2}{|c|}{-} & \multicolumn{2}{|c|}{-} & \multicolumn{2}{|c|}{136} \\
\hline \multicolumn{7}{|c|}{$\begin{array}{l}\text { TABLE 2: HYDRODYNAMIC DAMPING AND ADDED MAS } \\
\text { COEFFICIENTS FOR ANGULAR DIRECTION }\end{array}$} \\
\hline Descriptions & \multicolumn{2}{|c|}{ Roll } & \multicolumn{2}{|c|}{ Pitch } & \multicolumn{2}{|c|}{ Yaw } \\
\hline $\begin{array}{c}\text { CFD: } \\
\text { Damping } \\
\text { Coefficients }\end{array}$ & $K_{p}$ & $K_{p|p|}$ & $M_{q}$ & $M_{q|q|}$ & $N_{r}$ & $N_{r|r|}$ \\
\hline STAR CCM+ & 0.68 & 15.5 & 5.32 & 33.2 & 12.3 & 26.7 \\
\hline Experiment & - & - & - & - & 24.2 & 53.6 \\
\hline $\begin{array}{l}\text { CFD: Added } \\
\text { mass } \\
\text { coefficients }\end{array}$ & \multicolumn{2}{|c|}{$K_{\dot{p}}$} & \multicolumn{2}{|c|}{$M_{\dot{q}}$} & \multicolumn{2}{|c|}{$N_{\dot{r}}$} \\
\hline WAMIT & \multicolumn{2}{|c|}{2.80} & \multicolumn{2}{|c|}{13.7} & \multicolumn{2}{|c|}{5.26} \\
\hline Experiment & \multicolumn{2}{|c|}{-} & \multicolumn{2}{|c|}{-} & \multicolumn{2}{|c|}{4.30} \\
\hline
\end{tabular}

To validate the CFD results obtained in Table 1, the hydrodynamic parameters in the heave and yaw direction were tested in the water tank [10] as shown in Fig. 4 (right). To simplify the model, the ROV is not commanded to move in the angular direction during the test. Hence, the centripetal and Coriolis terms are not dominant [1] as the ROV is not performing the rotational motion. The effect of the quadratic components of the hydrodynamic damping is negligible for ROV moving at a slow speed of approximately $0.08 \mathrm{~m} / \mathrm{s}$. The resulted heave model [10] for ROV can be simplified to:

$$
\left(m+Z_{\dot{w}}\right) \dot{w}+Z_{w} w=\tau_{z}
$$

Rearranging the preceding equation in matrix form gives:

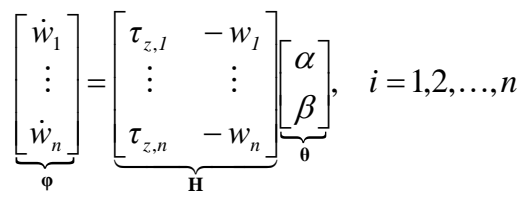

where $\alpha=1 /\left(m+Z_{\dot{w}}\right), \beta=-Z_{w} /\left(m+Z_{\dot{w}}\right)$ represent positive value, and $n$ represents the number of samples data from the experiment. The standard least square solution is provided by the Moore-Penrose pseudoinverse: $\widehat{\gamma}=\left(\mathbf{H}^{\mathrm{T}} \mathbf{H}\right)^{-1} \mathbf{H}^{\mathrm{T}} \boldsymbol{\varphi}$. The recursive least square (RLS) is applied to compute the $Z_{w}, Z_{\dot{w}}$ parameters of the ROV. The parameters update $\widehat{\gamma}_{\mathrm{RLS}}(t)$ for every time instant includes a correction term to the previous estimate $\hat{\gamma}_{\mathrm{RLS}}(t-1)$. Note that ROV model parameters are treated as time-invariant. The RLS needs an initial value of $\widehat{\gamma}_{\mathrm{RLS}}(t)$ and the error covariance matrix $\mathrm{P}$. The initial value of $\hat{\gamma}_{\mathrm{RLS}}(t)$ is $[0,0, \ldots, 0]^{\mathrm{T}}$ and $\mathrm{P}$ is $100 \mathbf{I}_{2}$ where $\mathbf{I}_{2}$ is the identity matrix of dimension equal to 2 . The forgetting factor is set to 0.001 . The total thrust used to move the ROV in heave direction is approximately $10 \mathrm{~N}$. The data was sampled at a frequency of $100 \mathrm{~Hz}$. The comparison between the RLS and measured results of the heave velocity was performed in the water tank. The rootmean-squared velocity error is approximately 0.044. As compared to the earlier CFD results in heave direction (see the last column of Table 1), there is approximately 50\% error in the damping coefficient while the added mass coefficient has around not more than $8 \%$. It could be due to the model inaccuracy caused by modeling and other uncertainties in measurements.

The following yaw model [10] for the ROV is validated.

$$
\left(I_{r}+N_{\dot{r}}\right) \dot{r}+N_{|r| r}|r| r+N_{r} r=\tau_{\psi}
$$

where $I_{r}$ is the moment of inertia and $N_{\dot{r}}$ is the added mass coefficient in the yaw direction. $N_{r}$ is the linear component of drag force and $N_{|r| r}$ is the quadratic component of drag force in the yaw direction. Here, $r$ is the yaw rate and $\tau_{\psi}$ is the moment input to ROV from the respective thrusters. With a constant heading velocity $(\dot{r}=0)$, the preceding equation [10] becomes:

$$
N_{|r| r}|r| r+N_{r} r=\tau_{\psi}
$$

The test was performed to estimate the drag coefficients $N_{|r| r}$ and $N_{r}$. A fixed velocity (corresponding voltage) commands were applied to the thrusters on the vehicle, and the steady state yaw rate was recorded. The data recorded consists of both linear and quadratic drag forces. The torque and corresponding steady state yaw rate are tabulated. The curve fitted values for $N_{|r| r}$ and $N_{r}$ in (18) are approximately 24.2 and 53.6, respectively. The added mass of ROV in the yaw direction was estimated using a sinusoidal control command applied to the thrusters and the yaw rate. A sinusoidal control signal with $10 \mathrm{~Hz}$ frequency was applied to the thrusters with a value ranging $+2.7 \mathrm{~V}$ to $-2.7 \mathrm{~V}$ in the forward and reverse directions, respectively. With $2.7 \mathrm{~V}$ constant control signal, the constant heading velocity is approximately $0.435 \mathrm{rad} / \mathrm{s}$. This value corresponds to 31.52 $\mathrm{Nm}$ to achieve the yaw rate of $0.435 \mathrm{rad} / \mathrm{s}$. However, the maximum sinusoidal angular speed is approximately 0.385 $\mathrm{rad} / \mathrm{s}$. As the measured yaw rate is smaller than the speed measured in the water test, it shows that the ROV needs an addition moment to overcome the resistance (i.e. added 
mass) due to its body mass and the added mass around the ROV. If the amplitude of sine wave is increased to the maximum heading velocity of $0.435 \mathrm{rad} / \mathrm{s}, \mathrm{a}+2.9 \mathrm{~V}$ to $-2.9 \mathrm{~V}$ sinusoidal control signal with moment approximately equal to $35.17 \mathrm{Nm}$ is required. It indicates an additional of 3.65 $\mathrm{Nm}$ was needed to overcome both the ROV's inertia and added mass during maneuvering. The maximum heading acceleration is approximately $0.85 \mathrm{rad} / \mathrm{s}^{2}$.

The moment due to the body inertia and added mass is computed as $\left(I_{r}+N_{\dot{r}}\right) \dot{r}=\Delta \tau_{\psi}=3.65 \mathrm{Nm}$. Substituting the peak acceleration of $0.85 \mathrm{rad} / \mathrm{s}^{2}$ and the added mass coefficient, $N_{\dot{r}}$ equals to $4.3 \mathrm{~kg} . \mathrm{m}^{2}$ was obtained. The hydrodynamic added mass and damping coefficients for the yaw direction are tabulated in Table 2. There is approximately $50 \%$ error in the damping coefficient while the error in the added mass coefficients is not exceeding $20 \%$.

The remaining parameters are not validated due to the limitations in the test facilities and resources. However, it is clear there exists an error of at least $20 \%$ in the hydrodynamic damping and added mass coefficient in both the heave and yaw model (and DoFs that are not measured). It creates some uncertainties in the modeling and measurement process.

\section{RoBUSt CONTROL SYSTEM DESIGN}

A robust sliding mode controller incorporating evolutionary fuzzy-based scheme is intended to tune the sliding-mode controller parameters despite the model uncertainties and the external disturbance. The external disturbances are usually bounded and attenuated by the controller. Recall the following definitions where $\mathbf{v}=\left[\begin{array}{llllll}u & v & w & p & q & r\end{array}\right]^{\mathrm{T}}$ is the velocity of the fluid in body-fixed coordinate, $\mathbf{v}_{\mathbf{r}}=\mathbf{v}-\mathbf{v}_{\mathbf{c}}$ is the relative velocity between the fluid and ROV. As fluid is assumed to be irrotational, the fluid velocities are given as follows.

$$
\mathbf{v}_{\mathbf{c}}=\left[\begin{array}{llll}
u_{c} & v_{c} & w_{c} & \mid 0_{1 \times 3}
\end{array}\right]^{\mathrm{T}}
$$

The ROV dynamics in [1] includes the current disturbance and model uncertainty in the following form.

$$
\mathbf{M} \dot{\mathbf{v}}+\mathbf{C}(\mathbf{v}) \mathbf{v}+\mathbf{D}\left(\mathbf{v}_{\mathbf{r}}\right)+\mathbf{H}\left(\mathbf{v}, \mathbf{v}_{\mathbf{c}}, \dot{\mathbf{v}}_{\mathbf{c}}\right)=\boldsymbol{\tau}
$$

where the term $\mathbf{H}\left(\mathbf{v}, \mathbf{v}_{\mathbf{c}}, \dot{\mathbf{v}}_{\mathbf{c}}\right)$ contains the hydrodynamic forces between the submerged ROV and the motion of the fluid. The term $\mathbf{D}\left(\mathbf{v}_{\mathbf{r}}\right)$ is the hydrodynamic damping force characterized as the sum of the linear and quadratic function of the relative velocity between the ROV and the fluid. Here, a known nominal model (denoted by subscript 'o') of the vehicle dynamics has the following form:

$$
\mathbf{M}_{\mathbf{o}} \dot{\mathbf{v}}+\mathbf{C}_{\mathbf{o}}(\mathbf{v}) \mathbf{v}+\mathbf{D}_{\mathbf{o}}\left(\mathbf{v}_{\mathbf{r}}\right)=\tau_{\mathrm{o}}
$$

Equation (20) is rewritten as:

$$
\mathbf{M}_{\mathbf{o}} \dot{\mathbf{v}}+\mathbf{C}_{\mathbf{o}}(\mathbf{v}) \mathbf{v}+\mathbf{D}_{\mathbf{o}}\left(\mathbf{v}_{\mathbf{r}}\right)+\Delta\left(\mathbf{v}, \mathbf{v}_{\mathbf{c}}, \dot{\mathbf{v}}_{\mathbf{c}}\right)=\boldsymbol{\tau}
$$

where $\mathbf{M}_{\mathbf{0}}$ is a diagonal matrix,

$$
\Delta\left(\mathbf{v}, \mathbf{v}_{\mathbf{c}}, \dot{\mathbf{v}}_{\mathbf{c}}\right)=\Delta \mathbf{M} \dot{\mathbf{v}}+\Delta \mathbf{C}(\mathbf{v}) \mathbf{v}+\Delta \mathbf{D}\left(\mathbf{v}_{\mathbf{r}}\right)+\mathbf{H}\left(\mathbf{v}, \mathbf{v}_{\mathbf{c}}, \dot{\mathbf{v}}_{\mathbf{c}}\right)
$$
and

$$
\Delta \mathbf{M}=\mathbf{M}-\mathbf{M}_{\mathbf{0}} ; \Delta \mathbf{C}=\mathbf{C}(\mathbf{v})-\mathbf{C}_{\mathbf{0}}(\mathbf{v}) ; \Delta \mathbf{D}=\mathbf{D}\left(\mathbf{v}_{\mathbf{r}}\right)-\mathbf{D}_{\mathbf{0}}\left(\mathbf{v}_{\mathbf{r}}\right)
$$

Note that the parameters used in (22) can be obtained from nominal model except for $\Delta\left(\mathbf{v}, \mathbf{v}_{\mathbf{c}}, \dot{\mathbf{v}}_{\mathbf{c}}\right)$ that defines the lumped uncertainty due to the model uncertainties and external underwater current is bounded by the following properties.

$$
\begin{gathered}
\exists m_{1}, m_{2}>0: m_{1}\|\mathbf{v}\|^{2} \leq \mathbf{v}^{\mathbf{T}} \mathbf{M}_{\mathbf{o}} \mathbf{v} \leq m_{2}\|\mathbf{v}\|^{2}, \forall \mathbf{v} \in \mathfrak{R}^{6} \\
\exists \mu_{1}>0: 0 \leq \mathbf{v}^{\mathbf{T}} \Delta \mathbf{M v} \leq \mu_{1}\|\mathbf{v}\|^{2} \\
\exists \mu_{2}>0:\|\Delta \mathbf{C}(\mathbf{v})\| \mathbf{v} \leq \mu_{2}\|\mathbf{v}\| \\
\exists d_{i}>0, i=1, \ldots 3:\left\|\mathbf{D}\left(\mathbf{v}_{\mathbf{r}}\right)\right\| \leq \sum_{i=1}^{3} d_{i}\|\mathbf{v}\|+\sum_{i=1}^{i} d_{i}\left\|\mathbf{v}_{c}\right\|^{i} \\
\exists \beta_{i}>0, i=1, \ldots 3:\|\mathbf{H}\| \leq \beta_{i}\|\mathbf{v}\|\left\|\mathbf{v}_{\mathbf{c}}\right\|+\beta_{i}\left\|\mathbf{v}_{\mathbf{c}}\right\|^{2}+\beta_{i}\left\|\dot{\mathbf{v}}_{\mathbf{c}}\right\|^{2}
\end{gathered}
$$

The disturbances are bounded signals, $\mathbf{u}_{\mathbf{c}} \in L_{[0, \infty)}^{\infty}, \dot{\mathbf{u}}_{\mathbf{c}} \in L_{[0, \infty)}^{\infty}$ for $\mathbf{v}_{\mathbf{c}}$ and $\dot{\mathbf{v}}_{\mathbf{c}}$, respectively. The estimated bounds for the model uncertainties are obtained from ROV physical characteristics and experimental tests that indicate around more than $20 \%$ error or uncertainties in the nominal model in Section II. Once the upper bound for $\mathbf{u}_{\mathbf{c}}$ and $\dot{\mathbf{u}}_{\mathbf{c}}$ is fixed, the effect of the disturbance becomes a bounded perturbation on the nominal ROV model.

\section{A. Sliding-Mode Control}

The mathematically proof for the stability and convergences of the SMC for the evolutionary fuzzy-based scheme is shown. In SMC, a sliding surface in term of error is defined. Any states that reach the sliding surface implies convergence to the desired earth-fixed coordinate defined as $\eta_{\mathbf{d}}=\left[\begin{array}{llllll}x_{d} & y_{d} & z_{d} & \phi_{d} & \theta_{d} & \psi_{d}\end{array}\right]^{\mathrm{T}}$.

Let the earth-fixed coordinate error denoted by $\widetilde{\boldsymbol{\eta}}=\boldsymbol{\eta}-\boldsymbol{\eta}_{\mathbf{d}}$ with the sliding surfaces expressed as:

$$
\mathbf{s}=\dot{\tilde{\boldsymbol{\eta}}}+\lambda \tilde{\boldsymbol{\eta}}
$$

where $\boldsymbol{\eta}=\left[\begin{array}{llllll}x & y & z & \phi & \theta & \psi\end{array}\right]^{\mathrm{T}}$ is the actual earthfixed coordinate and $\lambda>0$ is the bandwidth. A common Lyapunov function is written as such:

$$
V(t)=\frac{1}{2} \mathbf{s}^{\mathbf{T}} \mathbf{M}_{\mathbf{o}} \mathbf{s}
$$

The time derivative yields:

$$
\dot{V}(t)=\mathbf{s}^{\mathbf{T}} \mathbf{M}_{\mathbf{0}} \dot{\mathbf{s}}
$$

With $\quad \mathbf{M}_{\mathbf{o}} \dot{\mathbf{s}}=\mathbf{M}_{\mathbf{o}}(\ddot{\boldsymbol{\eta}}+\lambda \dot{\boldsymbol{\eta}}), \quad \ddot{\boldsymbol{\eta}}_{\mathbf{d}}=0, \dot{\boldsymbol{\eta}}_{\mathbf{d}}=0 \quad$ and

$\ddot{\boldsymbol{\eta}}=\mathbf{J}(\boldsymbol{\eta}) \dot{\mathbf{v}}+\dot{\mathbf{J}}(\boldsymbol{\eta}) \mathbf{v}, \dot{V}(t)$ can be written as:

$$
\begin{aligned}
\dot{V}(t)= & \mathbf{s}^{\mathbf{T}} \mathbf{M}_{\mathbf{o}} \mathbf{J M}_{\mathbf{o}}^{-\mathbf{1}}\left[\boldsymbol{\tau}-\mathbf{C}_{\mathbf{o}}(\mathbf{v}) \mathbf{v}-\mathbf{D}_{\mathbf{o}}\left(\mathbf{v}_{\mathbf{r}}\right)\right. \\
& \left.-\Delta\left(\mathbf{v}, \boldsymbol{\eta}, \mathbf{v}_{\mathbf{c}}, \dot{\mathbf{v}}_{\mathbf{c}}\right)\right]+\mathbf{s}^{\mathbf{T}} \mathbf{M}_{\mathbf{o}}(\dot{\mathbf{J}} \mathbf{v}+\lambda \dot{\boldsymbol{\eta}})
\end{aligned}
$$

Remark 1. The conditions $\ddot{\boldsymbol{\eta}}_{\mathbf{d}}=0$ and $\dot{\boldsymbol{\eta}}_{\mathbf{d}}=0$ for (33) imply that ROV is regulating its velocity around zero with almost zero acceleration for most of the time. In this case, the ROV is designed to maneuver slowly when it approaches its desired position.

From the properties in (25) to (29), there exist positive constant, $\varsigma_{1}, \varsigma_{2}$ and $\varsigma_{3}$ such that, $\Delta\left(\mathbf{v}, \mathbf{v}_{\mathbf{c}}, \dot{\mathbf{v}}_{\mathbf{c}}\right)$ in (23) is bounded

$$
\Delta\left(\mathbf{v}, \mathbf{v}_{\mathbf{c}}, \dot{\mathbf{v}}_{\mathbf{c}}\right) \leq \mu_{1}\|\dot{\mathbf{v}}\|+\varsigma_{1}\|\mathbf{v}\|+\varsigma_{2}\|\mathbf{v}\|^{2}+\varsigma_{3}=\delta(\mathbf{v}, t)
$$


The right-hand side of the inequality is a function (referred to $\delta(\mathbf{v}, t))$. It can be computed using the feedback law and the bounds established for the model uncertainties. The proposed control input $\boldsymbol{\tau}=\mathbf{T u}$ where the control input $\mathbf{u}$ can be written as:

$$
\begin{aligned}
\mathbf{u}=-\mathbf{T}^{+}\left\{\mathbf{M}_{\mathbf{o}} \mathbf{J}^{-1}[\right. & \left.K_{s p} \operatorname{sgn}(\mathbf{s})+K_{s i} \mathbf{s}+K_{s d} \mathbf{v}+\dot{\mathbf{J}} \mathbf{v}+\lambda \dot{\boldsymbol{\eta}}\right] \\
& \left.-\mathbf{C}_{\mathbf{o}}(\mathbf{v}) \mathbf{v}-\mathbf{D}_{\mathbf{o}}\left(\mathbf{v}_{\mathbf{r}}\right)-\delta(\mathbf{v}, t)\right\}
\end{aligned}
$$

where $K_{s p}, K_{s i}, K_{s d}$ are the adjustable SMC's gains and $\mathbf{T}^{+}=\left(\mathbf{T}^{\mathbf{T}} \mathbf{T}\right)^{-\mathbf{1}} \mathbf{T}^{\mathbf{T}}$ is the Moore-Penrose pseudoinverse. Substituting the control law back into $\dot{V}$ yields:

$$
\dot{V}(t) \leq \mathbf{s}^{\mathbf{T}} \mathbf{M}_{\mathbf{o}}\left[-K_{s p} \operatorname{sgn}(\mathbf{s})-K_{s i} \mathbf{s}-K_{s d} \mathbf{v}\right] \leq 0
$$

This is because the control gains $K_{s p}, K_{s i}$ and $K_{s d}$ are chosen to be large enough such that $\dot{V}(t) \leq 0$. Notice that, $\dot{V} \leq 0$ implies $V(t) \leq V(0)$, and therefore that $\mathbf{s}$ is bounded. This in turn implies that $\ddot{V}$ is bounded and $\dot{V}$ must be uniformly continuous. The application of Barbalat's lemma [26] then shows that $\mathbf{S} \rightarrow 0$ gives $\dot{\tilde{\boldsymbol{\eta}}}+\lambda \tilde{\boldsymbol{\eta}}=0$, and thus $\tilde{\boldsymbol{\eta}}(t)=e^{-\lambda t} \tilde{\boldsymbol{\eta}}(0)$ implies the position error, $\tilde{\boldsymbol{\eta}}(t)$ converge to zero exponentially as $t \rightarrow \infty$. Hence, the system is asymptotically stable.

Remark 2. If the bound is chosen to be too large, the sign function in (36) will cause chattering in the control signals. However, if it selected to be too small, the stability condition will not be satisfied and lead to the unstable control system.

To ensure the stability under the uncertainties and decrease the requirements of the parameters in the control system, a genetic algorithm with fuzzy inference mechanism are used in the following section. Within the boundary layer, the fuzzy logic is used to reduce the chattering phenomenon by smoothing the switch signal.

\section{B. Proposed Fuzzy-Based Genetic Algorithm for SMC}

The genetic algorithm is embedded in SMC to produce the optimal control effort. The coefficients of the crossover and mutation step are selected using fuzzy inference mechanics, to give the right evolutionary direction and the suitable evolutionary step. The proposed control architecture for tuning SMC is shown in Fig. 3. As shown in Fig. 3, there are three parts including SMC mentioned previously. In the genetic algorithm, each chromosome is a point in population which consists of a finite number of chromosome. The chromosome is chosen by an evolutionary mechanism to obtain the fitness value. New population will form, and some members of the new population will produce new solutions. After some generations, the fittest control signal can be achieved. To decide on the correct evolutionary direction and stability in the system dynamic, the fuzzybased evolutionary is used. The fuzzy logic is also used to compensate for the chattering phenomenon inherent in the SMC with less discontinuous switching across.

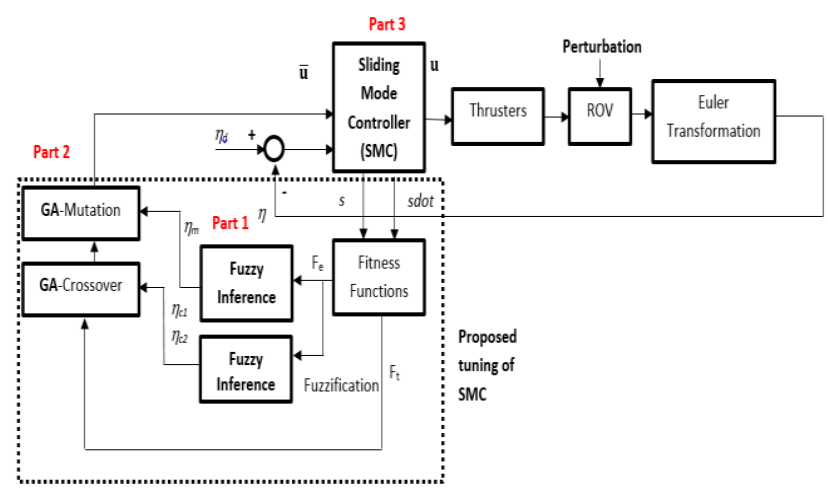

Fig. 3. Proposed control architecture for tuning sliding-mode controller

The ultimate evolutionary target of GA can be regarded as obtaining $\mathbf{s}(t)=0$ and $\dot{\mathbf{s}}(t)=0$ (i.e. the desired position is achieved). A fitness function used for tuning the coefficients in crossover and mutation for fuzzy interference is defined as

$$
\mathbf{F}_{\mathbf{e}}(\mathbf{s})=e^{-\sigma\left(\mathbf{s}^{2}+\dot{\mathbf{s}}^{2}\right)} \in[0,1]
$$

where $\mathbf{S}$ is a sliding surface defined in (30) and $\sigma$ is the rate of decay. The basic genetic operation is shown below.

1) Elites Selection in GA: The control voltage is treated as the chromosome in GA. The reproduction is utilized to decide the chromosomes into the mating pool for subsequent genetic operations. Equation (37) is used to decide the chromosomes that will be selected from the mating pool for further genetic operations. SMC's gains $\left(K_{s p}, K_{s i}, K_{s d}\right)$ for (35) are randomly selected from the positive operational region that ranges from $K_{\min }=0$ to $K_{\max }=50$. The sliding surface $\mathbf{S}$ and its derivative $\dot{\mathbf{S}}$ are used to compute the fitness value in (37). If the fitness of the new control input is greater than all previous values, it will be the new elite. The highest fitness value is regarded as the elite. By utilizing the elite as a parent, the generated offspring is the new control signal in the next control interval. If the fitness value of the new control signal is greater than the previous one, it will become the next elite. Therefore, it adopts the survival of the fittest.

2) Fuzzy-based Crossover and Mutation in GA: Crossover operation is the primary method to create perturbations in GA. It can generate offspring by varying the signature of the parent. The direction-based crossover operation is used in this paper. The concept is that the greater or further away from the sliding surface and its derivative, the greater the variation (or the control input) between the offspring and the parent. Conversely, the control input will be less or remain constant if the system dynamic is within the sliding surface. Hence, the sliding surface $\mathbf{s}$ and its derivative $\dot{\mathbf{S}}$ are integrated into the crossover operation. The offspring generated from the parent can be expressed as shown

$$
\mathbf{u}_{\text {GA,new }}=\mathbf{u}_{\text {GA,old }}+\left|\eta_{c 1}\right| \mathbf{s}+\left|\eta_{c 2}\right| \dot{\mathbf{s}}
$$

where $\mathbf{u}_{\mathbf{G A} \text {,old }}$ is the selected chromosome of $K_{s p}, K_{s i}, K_{s d}$ from the previous generation, $\mathbf{u}_{\mathbf{G A} \text {,new }}$ is the newly created offspring using $\eta_{c 1}$ and $\eta_{c 2}$ for $\mathbf{s}(t)$ and $\dot{\mathbf{s}}(t)$, respectively. The tuning coefficients $\eta_{c l}$ and $\eta_{c 2}$ are vital for crossover operation. For example, the tuning coefficients are chosen too large (small), the tuning step from generation to generation will be large (small) to enable 
the system dynamic to divergence quickly (become unstable). In this paper, the fuzzy crossover inference mechanism is used to select the right tuning coefficients.

The mutation in GA is then used to prevent the issue of the local minimal. A new chromosome can be included in the population. As compared to the current random mutation operation, there is no assurance that the offspring perform better than their parents that may affect the system dynamics performance in ROV. Hence, the direction-based mutation operation is used. The idea is that the mutation will be adjusted according to the gap between the sliding surface $(\mathbf{s}=0)$ and the system dynamics. If the control effort is unable to achieve close to the sliding surface, the mutation will force the ROV's dynamic to maintain nearer to the sliding surface. The offspring after mutation operation can be expressed as:

$$
\overline{\mathbf{u}}=\mathbf{u}_{\text {GA,new }}+\left|\eta_{m}\right|
$$

where $\eta_{m}$ is the adjustment of mutation operation from the fuzzy inference mechanism and $\overline{\mathbf{u}}$ is the offspring after mutation operation.

The occurrence of mutation operation in GA is performed using the mutation probability that may affect the system stability. Hence, a specified positive fitness value (set by the user) named FIT is utilized to check whether the mutation has occurred or not. If the fitness value is less than FIT, the mutation occurs. On the other hand, if the FIT is more than the specified value, the mutation will not happen. Also, the robustness of the GA is ensured by introducing the incremental uncertainties on the ROV model and external disturbance during the GA operation. In summary, the proposed Fuzzy-Based Genetic Algorithm on SMC (FGA_SMC) can be written in the following processes.

Step 1: Establish fitness function in (37).

Step 2: Choose from the initial population $\left(K_{s p}, K_{s i}, K_{s d}\right)$ for (35).

Step 3: Increment the uncertainty (up to $20 \%$ of the nominal ROV model and $1 \mathrm{~m} / \mathrm{s}$ of underwater current disturbance)

Step 4: Evaluate fitness value in (37) and select the elite $\mathbf{u}_{\text {GA,old }}$.

Step 5: Start fuzzy crossover operation to produce $\mathbf{u}_{\text {GA,new }}$ in (38).

Step 6: If the fitness value is less than FIT, proceed to next process or go to Step 8.

Step 7: Start fuzzy mutation operation to produce $\overline{\mathbf{u}}$ in (39).

Step 8: Compute control signal $\mathbf{u}$ in (35). It will be treated as the parent of the next generation as it is the most appropriate chromosome for the current situation.

The fuzzy inference mechanism is used in the evolutionary procedure. It also decides how to tune the above coefficients $\left(\eta_{c 1}, \eta_{c 2}, \eta_{m}\right)$ and the adjustment of mutation operation. The input variables are: B (Big), MI (Middle), S(Small). The output variables are: $\mathrm{H}$ (High), ME (Medium), L(Low). For fuzzy crossover, $\mathrm{X}$ represents $\mathbf{F}_{\mathbf{e}}(\mathbf{s})$, and $\mathrm{Y}$ represents the coefficients $\left(\eta_{c 1}, \eta_{c 2}\right)$ as seen in Fig. 3. For fuzzy mutation, $\mathrm{X}$ represents the sliding surface and $\mathrm{Y}$ represents the coefficients $\eta_{m}$. According to the process stated in Fuzzy-Based Genetic Algorithm (FGA), the evolutionary procedure comprising IF-THEN rules are as follows.

1) Rule 1 IF $X$ is B; THEN $Y$ is $L$;

2) Rule 2: IF $X$ is MI, THEN $Y$ is ME;

3) Rule 3: IF $X$ is $S$, THEN Y is $H$.

The computational effective triangular membership functions for each input and Singleton membership functions for the output (using product inference and average center defuzzifier) are used. The membership functions of the input and output fuzzy sets have $x_{1}, x_{2}$ and $x_{3}$ as the centers of the membership functions S, MI and B; $y_{1}, y_{2}$ and $y_{3}$ are the centers of the membership functions $\mathrm{L}$, $\mathrm{ME}$ and $\mathrm{H}$. The fuzzy inference mechanism output is then derived as shown.

$$
\bar{y}=\frac{w_{1} y_{3}+w_{2} y_{2}+w_{3} y_{1}}{w_{1}+w_{2}+w_{3}}=w_{1} y_{3}+w_{2} y_{2}+w_{3} y_{1}
$$

where $0 \leq w_{1} \leq 1,0 \leq w_{2} \leq 1,0 \leq w_{3} \leq 1$ are the weight of Rule 1, 2 and 3, respectively. The relation $w_{1}+w_{2}+w_{3}=1$ is valid according to the triangular membership function. Besides, the input and output fuzzy sets for the three parameters $\left(\eta_{c 1}, \eta_{c 2}, n_{m}\right)$ are different. They can be determined by similar fuzzy rule and inference mechanism in (40).

\section{SIMULATION AND EXPERIMENTAL RESULTS}

\section{A. ROV System Design}

The ROV in Fig. 4 has an open-frame design and is $1.5 \mathrm{~m}$ long, $1 \mathrm{~m}$ wide, and $1.7 \mathrm{~m}$ high. It has a dry weight of around $135 \mathrm{~kg}$ and a working depth of at least $100 \mathrm{~m}$. It is designed for launch and recovery of an AUV. The vehicle is installed with six electric brushless thrusters. The roll and pitch motion is not controlled as the metacentric height is quite large to give good static stability.

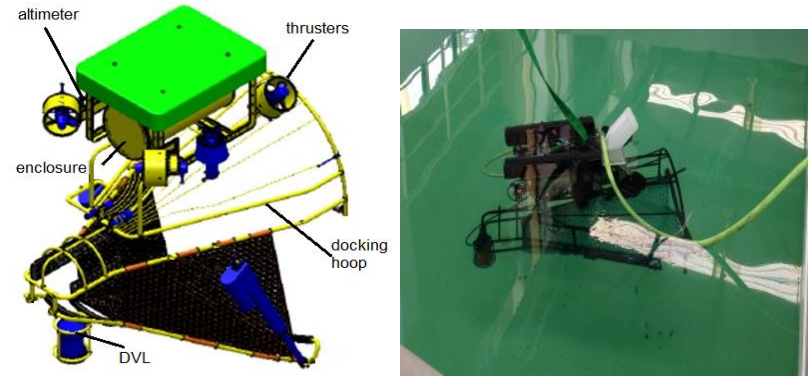

Fig. 4. ROV prototype (left) and actual design (right) in water tank

The following sensor and actuators are connected to a target PC using Diamond Systems ATHENA III PC104 operating in Ubuntu platform as shown in Fig.4. The main components used are as follows.

1) NavQuest Doppler Velocity Log (DVL) to measure the velocity of $R O V$.

2) Druck pressure sensor or altimeter is used to measure the depth of the ROV;

3) XSENS inertia measurement unit (IMU) is used to measure the positions and angular positions of the ROV; 4) two USB Cameras for underwater image capturing; USB Joystick for remote control; 
5) six DC Tecnadyne brushless electric thrusters connected to an ISO-6 thruster driver card to control the speed of the propellers.

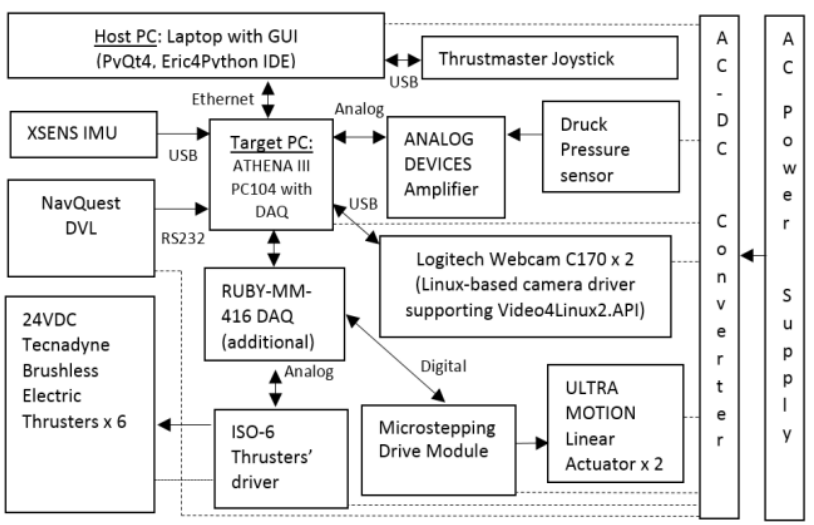

Fig. 5. System architecture design for ROV

In Fig. 5, the host PC with a graphical user interface developed by open-source platform using Python-based IDE as the integrated development environment to communicate with ROV via an Ethernet connection. The remaining components are housed in an aluminum enclosure with openings for subsea electrical connectors. ROV has an umbilical cable linked to the surface vessel for communication, and at the same time to provide the power (230V AC power) for the vehicle. The cage with a net (see Fig.4) attached to the bottom of ROV provides a docking hoop for holding an AUV during the launching and recovering operation. ROV needs to position itself at a predetermined target near the aft of the vessel as shown in Fig.6. During the mission, ROV will be launched from the surface vessel. The operator onboard the vessel moves the ROV remotely from the vessel to the vicinity of the desired target point (see position 1 in Fig. 6). At this point, the ROV will maneuver autonomously to the final desired depth and heading angle (see position 2 in Fig. 6) to launch AUV, and station-keeping at the same location to recover AUV after the mission. When ROV returns to position 1, ROV and AUV will be remotely recovered to the launch vessel.

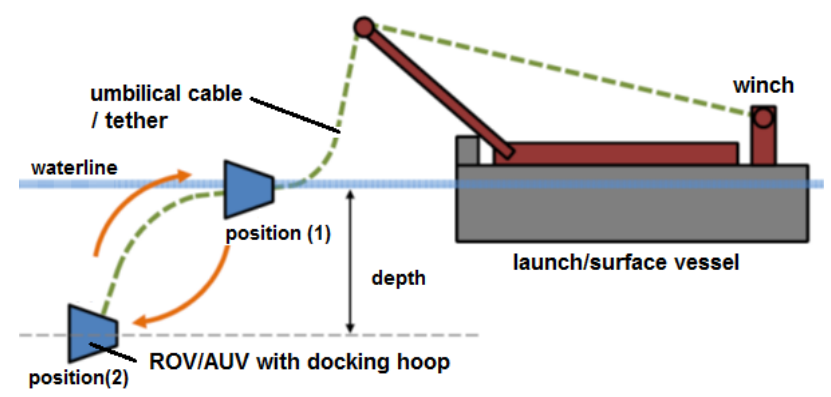

Fig. 6. Schematic of ROV launching (position 1) and recovery (position 2)

\section{B. ROV Simulation}

In the simulation, the ROV was controlled to move diagonally in the horizontal plane from its zero initial position to $\left(x_{d}=y_{d}=0.5 \mathrm{~m}\right)$ as shown in the position (1) in earlier Fig. 6 . The controller gains $\left(K_{s p}, K_{s i}, K_{s d}\right)$ used for the ROV control are tuned using the proposed tuning approach. The outputs of the FLC are namely: $\eta_{c 1} \quad \eta_{c 2}$ and $\eta_{m}$. The nominal ROV model can be obtained from the Table 1. For comparison; there are two simulation cases.
Case 1: Simulate the proposed FGA_SMC is first carried out at the different perturbation level. The control system is tested up to $20 \%$ (or 1.2 times the nominal ROV model) under the current underwater speed of $1 \mathrm{~m} / \mathrm{s}$.

Case 2: Compare the proposed method with SMC, fuzzy logic and PID at $20 \%$ perturbation on the ROV model with the underwater current speed to $1 \mathrm{~m} / \mathrm{s}$.

The following two cases are carried out in MATLAB/Simulink package, and their control parameters are given below.

1) Proportional-Integral-Derivative (PID)

The resulted PID controller gains was automatically tuned PID Tuner (in MATLAB/Simulink) to meet the steady-state value with small overshoot, short settling time, short rise time, higher gain and phase margin for closed-loop stability. The PID gains are as follows.

$$
K_{p}=14, K_{i}=5, K_{d}=15
$$

2) Conventional Sliding-Mode Controller(SMC)

The conventional SMC parameters used are as follows: $K_{s p}$ $=26, K_{s i}=42, K_{s d}=37, \lambda=0.01$

3) Fuzzy logic Controller

The parameters of fuzzy logic controller without the proposed fuzzy-based evolutionary process are $\eta_{c 1}=\eta_{c 2}=1$ with random mutation.

4) Proposed Fuzzy-Based Genetic Algorithm for SMC (FGA_SMC)

The maximum number of generations is 100 , and the population size is 50 . As the aim is to cause the population to convergence, crossover (for convergence operation) should occur more frequently than the mutation (for divergence operation). But choosing the right probability can be quite subjective in different applications. Hence, a balanced approach of setting both the crossover and mutation probability to $20 \%$ was adopted in this paper. The fitness chromosome will be retained and duplicated in each generation. The SMC's gain distribution across the entire generation consists of 5000 points (i.e. 100 generations $\times 50$ populations). The lowest error norm (or highest fitness, $\left.\mathbf{F}_{\mathbf{t}}(t)\right)$ is at $96^{\text {th }}$ generation. The final fittest control parameters and variables used in FGA-SMC are as follows.

$$
\begin{array}{r}
K_{s p}=21, K_{s i}=5.9, K_{s d}=15, \sigma=0.1, \lambda=0.01, K_{\min }=0, \\
K_{\max }=50, \text { FIT }=0.3
\end{array}
$$

The output and input parameters used in the fuzzy-based evolutionary process are selected as shown.

$$
\begin{array}{r}
y_{1,1}=y_{1,2}=0.3, y_{2,1}=y_{2,2}=0.6, y_{3,1}=y_{3,2}=1 \\
y_{1, m}=-1, y_{2, m}=0, y_{3, m}=1 \\
x_{1,1}=x_{1,2}=0, x_{2,1}=x_{2,2}=0.5, x_{3,1}=x_{3,2}=1 \\
x_{1, m}=-0.5, x_{2, m}=0, x_{3, m}=0.5
\end{array}
$$

In Case 1, the simulation results of FGA-SMC can be seen in Fig. 7. The position and velocity time responses at different perturbed condition are plotted. The position responses indicate quite robust behavior to changing perturbations except for heave, roll, pitch, and yaw angle at higher perturbation. As the limit of the uncertainty increase to more than $8 \%$ and underwater current disturbance greater than 1m/s, FGA_SMC continue to show quite robust performance with little change in the output response. The velocity responses also reflect a good regulation about zero velocities. The proposed control is robust only in low perturbations while in higher perturbation, only two of the six DoF display behavior of robustness to uncertainties. 

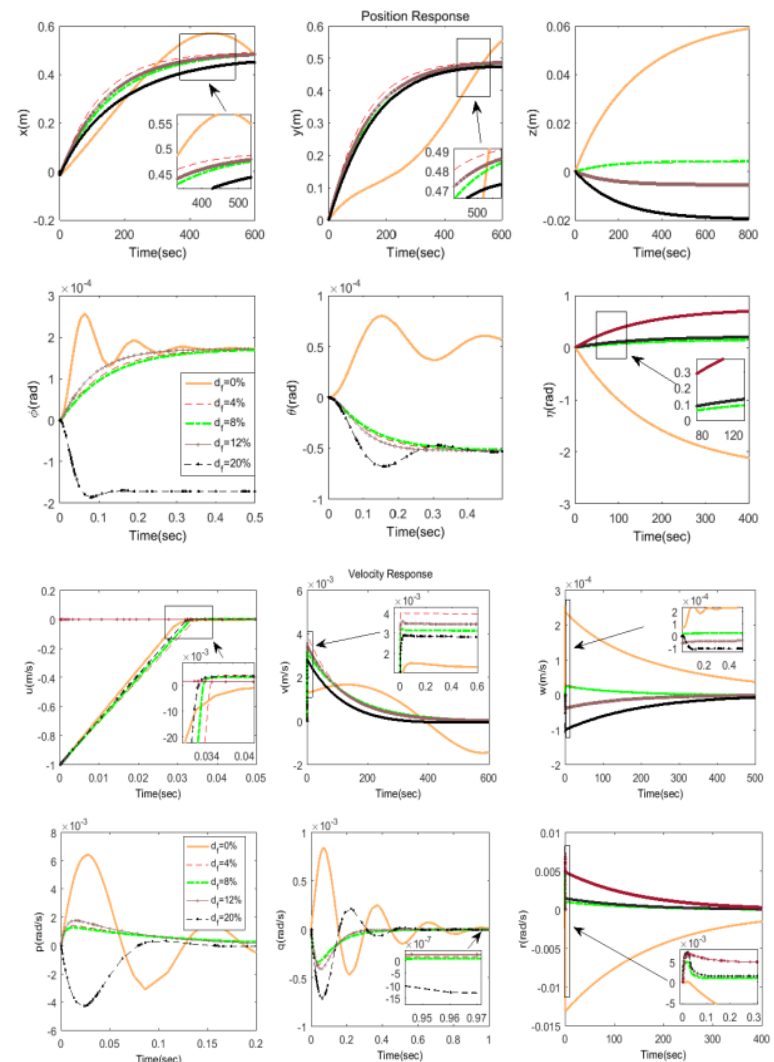

Fig. 7. Case 1: time-domain plots of position (top) and velocity (bottom) at various perturbed conditions $\left(x_{d}=y_{d}=0.5 \mathrm{~m}\right)$ using FGA-SMC

In Case 2, the controller parameters are used for the computer simulation to compare with other controllers (see Fig. 8) at $20 \%$ and $1 \mathrm{~m} / \mathrm{s}$. The proposed FGA-SMC controller exhibits a smaller error in the surge and sway position (where the set point is: $x_{d}=y_{d}=0.5 \mathrm{~m}$ ) than PID, SMC, and fuzzy-logic controller. The PID has a larger steady error as compared to all controllers. On the other hand, FGA-SMC exhibits less chattering phenomena as compared to SMC due to the fuzzy inference mechanism used in the evolutionary procedure. The fuzzy logic controller can compensate for the chattering phenomenon inherent in the conventional SMC. It resulted in less oscillatory behavior with a higher error in yaw position. On the other hand, SMC shows the inherent oscillatory behavior in all DoFs. As observed, the pitch and roll angle is small and quite self-stabilizing as compared to PID.

In summary, SMC, PID, and fuzzy-logic controller exhibit less robustness to the perturbation as seen in the overshoot, steady-state error and oscillatory responses as compared to the proposed FGA-SMC approach. The 2-norm position error also shows FGA-SMC has the lowest error as compared to the other controllers (FGA_SMC: 200; SMC: 600; PID: 1500; Fuzzy: 500). It implies that FGA-SMC proposed on ROV can handle the pre-determined uncertainty to achieve the robustness in the position control.
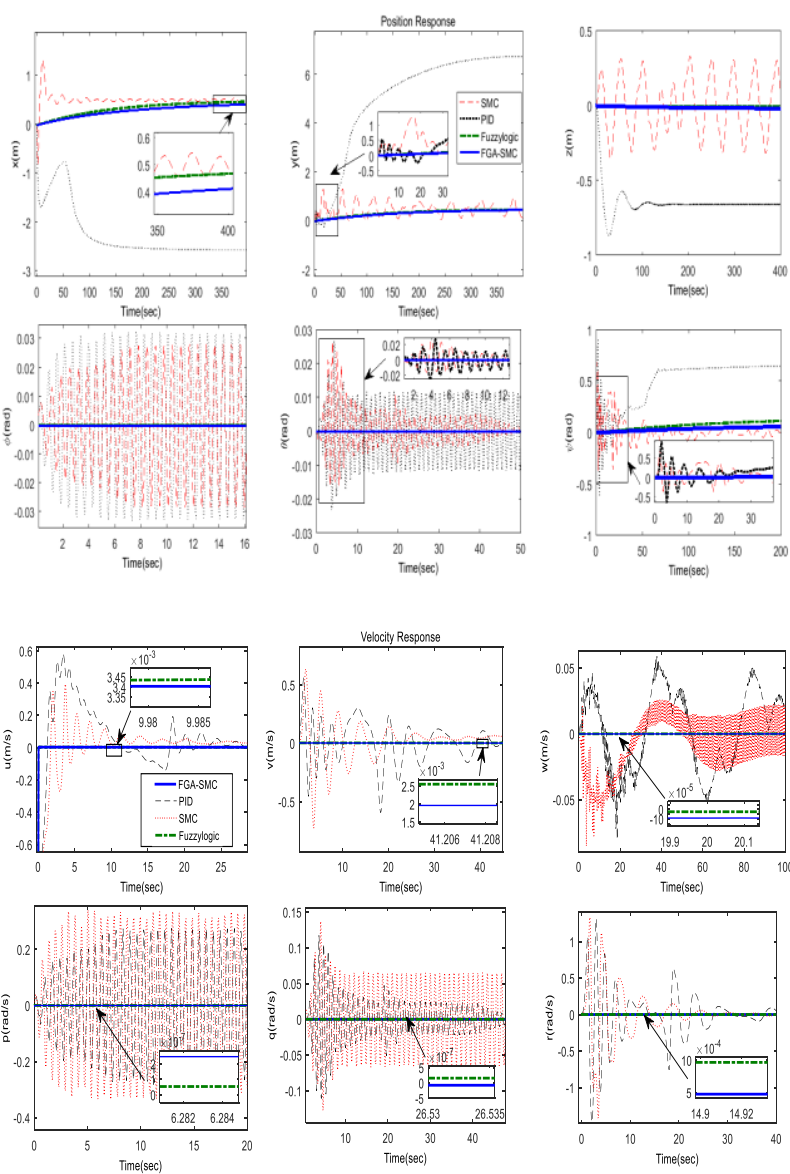

Fig. 8. Case 2: controller comparison of position (top) and velocity (bottom) performance at $20 \%$ model perturbation and $1 \mathrm{~m} / \mathrm{s}$ underwater current

\section{ROV Sea Trial}

The experimental test of ROV (see Fig. 9) was conducted near Singapore Yacht Club. The joystick was first used to move the ROV from a launch position (as shown earlier in Fig. 6) to position (1). At position (2), the following two tests namely: heave and yaw test were performed. The command signal and controller parameters were initialized using the graphical-user-interface designed by Python-Qt open-source codes. All data were sampled at $100 \mathrm{~Hz}$. Due to time constraint, FGA-SMC and PID controller were programmed into the target PC104 in ROV. During the experimental tests, the control parameters of FGA-SMC was further tuned to give a better response in real-time. It is attributed to the uncertain external factors such as different significant wave height (approximately $1 \mathrm{~m}$ ) and sea current (around 2 knots) from Meteorological Service Singapore affects the results and performance.

At position (2), the actual ROV was trying to regulate its depth of $0.6 \mathrm{~m}$ as shown in Fig. 9 (right). In the experiment, the original PID controller was unable to attenuate the disturbances. PID controller parameters was re-tuned to $K_{p}=31, K_{i}=27, K_{d}=34$. It was then used to compare with FGA-SMC. The controller gains used was tuned online with $K_{s p}=26, K_{s i}=11, K_{s d}=20$ and other parameters remain. As seen in Fig. 10 (top), PID controller, is unable to attenuate the disturbances due to the external wave and current on the ROV. PID controller tends to follow the wave perturbation. However, the FGA-SMC exhibits less oscillation with a small steady-state heave error of approximately $0.7 \%$, and the chattering response is much alleviated. FGA-SMC 
produces much smaller fluctuation in the yaw angle (except during the transient stage) as compared to PID controller during heave test. Similar behavior can be seen in the roll and pitch angle where less overshoot was observed in FGASMC than PID controller in Fig. 11. The results showed that FGA-SMC could robustly position the ROV at the desired depth despite the uncertainties and the external disturbances.

After the depth control, the results of the yaw control (see Fig. 10 (bottom)) were compared at 45 degrees. The yaw angle of the FGA-SMC settles to the steady-state value with a slow response (a similar behavior as seen during the simulation). Despite increasing the proportional gain, PID controller still exhibits an error of 15 degrees in the yaw angle. Although PID has a faster rise and settling time, FGA-SMC fairs better in term of the steady-state yaw angle despite its sluggish response. The sea trial reveals that ROV could maintain the desired yaw angle and the depth despite the model uncertainties and the external disturbances.

In summary, the proposed tuning of FGA-SMC is first applied on ROV to achieve a more efficient, and systematic architecture for SMC's tuning and better time-domain responses for deployment. With SMC integrated into GA on a real ROV platform with acceptable position responses with less chattering despite the sluggish response. As observed in the experimental results, the steady-state error in these results is attributed to the factor due to the changing uncertainties in an uncertain environment. Note that additional sensor such as surface acoustic wave sensor will be useful to provide measurable input to the controller. As shown in the captured response, the performance of the FGA-SMC designed controller is still better to that of the manually tuned system, in terms of steady-state error, less chattering behavior and robustness.
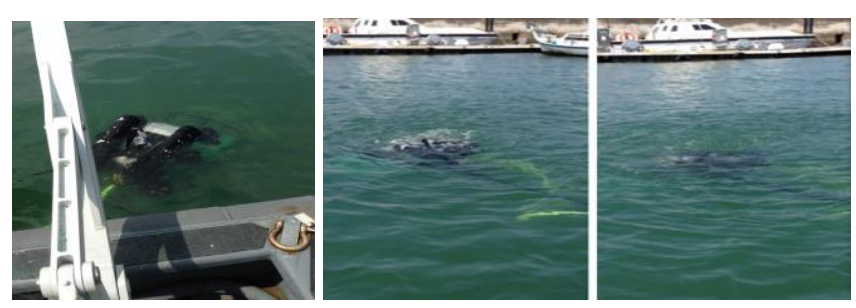

Fig. 9. ROV at launch position (left), yaw (middle) and heave (right) tests at position (2) using FGA-SMC

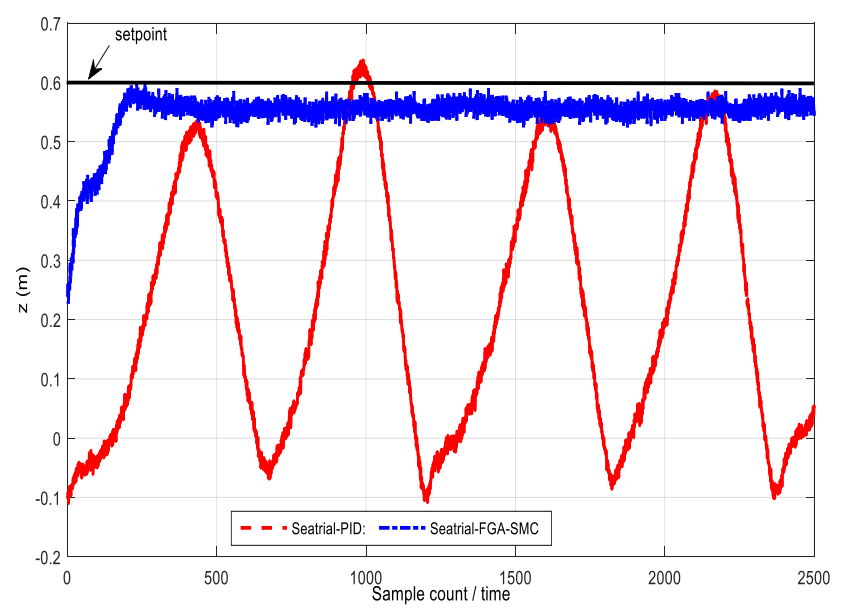

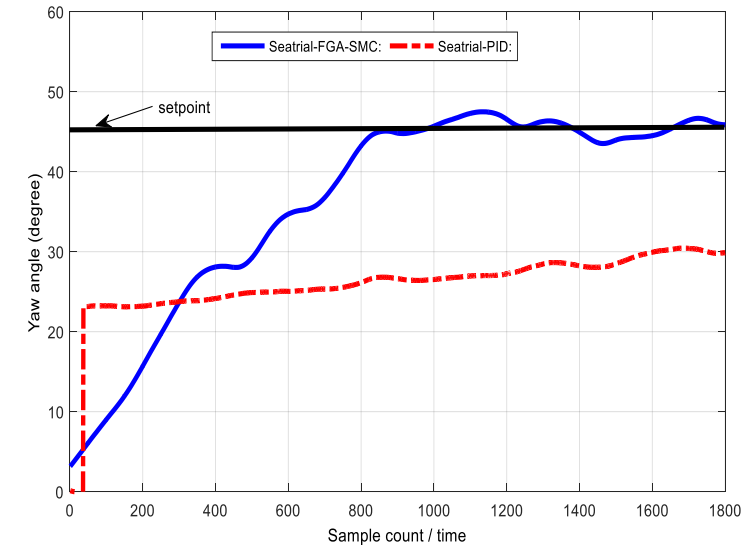

Fig. 10. Heave (top) and yaw (bottom) response between PID and FGASMC
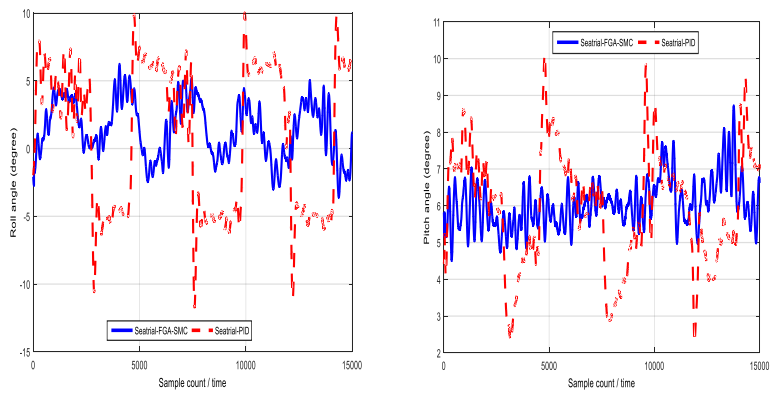

Fig. 11. Roll (left) and pitch (right) angle response between PID and FGASMC

\section{CONCLUSION}

The sliding-mode control (SMC) system using a direction-based genetic algorithm (GA) and fuzzy inference mechanism to influence the right evolutionary step and direction of the sliding mode control parameters was applied on an uncertain ROV system. The numerical simulation of ROV as compared to conventional SMC, PID, and fuzzy logic controller show its position stability and demonstrate its effectiveness under the different model and external uncertainties. As shown in the sea trial, the proposed fuzzy-based genetic algorithm for SMC are capable of controlling ROV to certain depth and yaw angle despite the uncertainties presence in the numerical model and the external operating environment. The main contributions of this study are summarized as follows. 1) The integration of inaccurate ROV model obtained by computational fluid dynamics for SMC system design. 2) The use of robust SMC with the fuzzy-based evolutionary procedure on an actual model subjected to model perturbation and external uncertainties in the evolutionary process. 3) SMC was integrated into GA to develop a real-time code GA control systems on a real ROV platform with good position responses with less chattering. For future works, more control techniques with acoustic wave sensor will be incorporated on multiple ROVs. The Type 2-Fuzzy Logic controller to better cope with modeling uncertainties will be used in the ROV. 


\section{ACKNOWLEDGMENT}

We want to acknowledge Newcastle University (Mr. Junyi Lin and other staff members) and ST Electronics for providing fund (KH134514/EQS0459/28) and staff (Mr Kum Hoong Cheong, Mr Leonard Looi, Mr Lim JunJie, Mr Elvin Teh and other staff members) for testing, work place, parts, manpower and mechanical design.

\section{REFERENCES}

[1] T. I. Fossen, Marine Control Systems: Guidance, Navigation, and Control of Ships, Rig and Underwater Vehicles, Marine Cybernetics Trondheim, 2002.

[2] J.H. Kim; J.C. Lee and Y.R. Choi, "LAROB: Laser-Guided Underwater Mobile Robot for Reactor Vessel Inspection," IEEE/ASME Transactions on Mechatronics, vol. 19, no. 4, pp. 1216 - 1225, 2014.

[3] O. Chocron, U. Prieur and L. Pino. "A Validated Feasibility Prototype for AUV Reconfigurable Magnetic Coupling Thruster," IEEE/ASME Transactions on Mechatronics, vol.19, no. 2 , pp. 642 - 650, 2014.

[4] C. Silvestre, R. Cunha, N. Paulino and A. Pascoal, "A BottomFollowing Preview Controller for Autonomous Underwater Vehicles," IEEE Transactions on Control Systems Technology, vol. 17, no. 2, pp. 257 266, 2009.

[5] A. Mazumdar, M.S. Triantafyllou and H.H. Asada, "Dynamic Analysis and Design of Spheroidal Underwater Robots for Precision Multidirectional Maneuvering," IEEE/ASME Transactions on Mechatronics, vol. 20, no. 6, pp. 2890 - 2902, 2015.

[6]A. Tyagi and D. Sen, "Calculation of transverse hydrodynamic coefficients using computational fluid dynamic approach," Ocean Engineering, vol. 33, no. 5, pp. 798-809, 2006.

[7] J.L.D. Dantas, E.A. de Barros, "Numerical analysis of control surface effects on AUV manoeuvrability," Applied Ocean Research, vol. 42, pp.168-181,2013

[8] M. Caccia, G. Indiveri and G. Veruggio, "Modeling and identification of openframe variable configuration unmanned underwater vehicles," IEEE J. Ocean. Eng., vol. 25, pp. 227- 240, 2000.

[9] W. L. Chan and T. Kang, "Simultaneous Determination of Drag Coefficient and Added Mass," IEEE Journal of Oceanic Engineering, vol. 36, no. 3, pp. 422-430, 2011.

[10] CS. Chin, WP. Lin, JY. Lin, "Experimental validation of open-frame ROV model for virtual reality simulation and control," Journal of Marine Science and Technology, pp 1-21, 2017.

[11] H. Zhang, G. Zhang and J. Wang, "H infinity observer design for LPV systems with uncertain measurements on scheduling variables: Application to an Electric Ground Vehicle," IEEE/ASME Transactions on Mechatronics, vol. 21, no. 3, pp. 1659 - 1670, 2016.

[12] Q. Meng, T.Zhang, X. Gao and J. Song, "Adaptive sliding mode fault-tolerant control of the uncertain Stewart platform based on offline multibody dynamics," IEEE/ASME Transactions on Mechatronics, vol. 19, no. 3, pp. $882-894,2014$.

[13]R. Cui, L. Chen, C. Yang, M. Chen, "Extended state observer-based integral sliding mode control for an underwater robot with unknown disturbances and uncertain nonlinearities," IEEE Transactions on Industrial Electronics, vol. 64, no. 8, pp.6785-6795.

[14] C.J. Lin, H.T. Yau, Y.C. Tian, "Identification and Compensation of Nonlinear Friction Characteristics and Precision Control for a Linear Motor Stage," IEEE/ASME Transactions on Mechatronics, vol. 18, no. 4, pp. 1385-1396, 2013.

[15] J. Shen; Q. Lu; C. Wu; W. Jywe, "Sliding-Mode Tracking Control With DNLRX Model-Based Friction Compensation for the Precision Stage," IEEE/ASME Transactions on Mechatronics, vol. 19, no.2, pp. 788 $-797,2014$.

[16] S. Perić, D. Antić, M. Milovanović, D. Mitić, M. Milojković and S. Nikolić, "Quasi-Sliding Mode Control With Orthogonal Endocrine Neural Network-Based Estimator Applied in Anti-Lock Braking System," IEEE/ASME Transactions on Mechatronics, vol. 21, no. 2, pp. 754 - 764 2016.

[17] RJ. Wai and R.Muthusamy, "Fuzzy-Neural-Network Inherited Sliding-Mode Control for Robot Manipulator Including Actuator Dynamics," IEEE Transactions on Neural Networks and Learning Systems, vol.24, no. 2, pp. $274-287,2013$.

[18] E. Kayacan, E. Kayacan; H. Ramon, O. Kaynak and W. Saeys , "Towards Agrobots: Trajectory Control of an Autonomous Tractor Using Type-2 Fuzzy Logic Controllers," IEEE/ASME Transactions on Mechatronics, vol. 20, no. 1, pp. 287 - 298, 2015.
[19] R. Nair, L. Behera, V. Kumar and M. Jamshidi, "Multisatellite Formation Control for Remote Sensing Applications Using Artificial Potential Field and Adaptive Fuzzy Sliding Mode Control," IEEE Systems Journal, vol. 9, no. 2, pp. 508-518, 2015

[20] Y. Du, J. Fang and C. Miao, "Frequency-Domain System Identification of an Unmanned Helicopter Based on an Adaptive Genetic Algorithm," IEEE Transactions on Industrial Electronics, vol. 61, no. 2, pp. 870-881, 2014.

[21] L. Yao, K.S. Weng and M.S. Wu, "Evolutionary Learning of Classifiers for Disk Discrimination," IEEE/ASME Transactions on Mechatronics, vol. 20, no. 6 ,pp. 3194 - 3203, 2015.

[22]H. K. Lam, F. H. Leung, and P. K. S. Tam, "Design and stability analysis of fuzzy model-based nonlinear controller for nonlinear systems using genetic algorithm," IEEE Trans. Syst., Man, Cybern. B, vol. 33, no. 2, pp. 250-257, Apr. 2003.

[23] F. H. F. Leung, H. K. Lam, S. H. Ling, and P. K. S. Tam, "Optimal and stable fuzzy controllers for nonlinear systems based on an improved genetic algorithm," IEEE Trans. Ind. Electron., vol. 51, no. 1, pp. 172182, Feb. 2004.

[24] F. H. F. Leung, H. K. Lam, S. H. Ling, and P. K. S. Tam, "Tuning of the structure and parameters of neural network using an improved genetic algorithm," IEEE Trans. Neural Networks, vol. 14, pp. 79-88, Jan. 2003. [25]WP. Lin, CS. Chin, LCW Looi, JJ Lim, EME Teh, "Robust Design of Autonomous Underwater Vehicle Docking Hoop for Recovery Operation," Robotics, 4(4), pp. 492-515, 2015.

[26] J.-J. E. Slotine and W. Li, Applied Nonlinear Control, 1st ed. Englewood Cliffs, NJ, USA: Prentice-Hall, 1991 